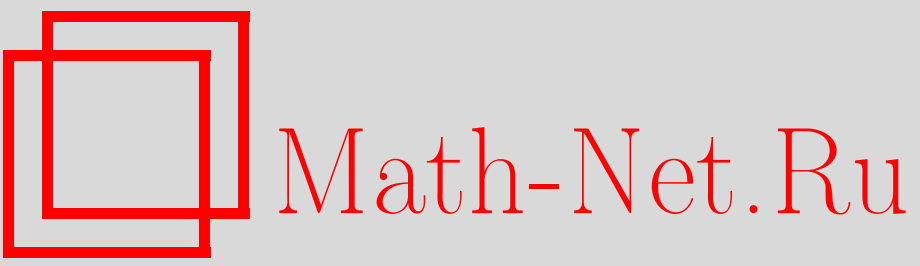

В. Ф. Ковалев, Ренормгрупповой анализ сингулярности в задаче о самофокусировке волнового пучка, ТМФ, 1999, том 119, номер 3, 405-418

DOI: https://doi.org/10.4213/tmf748

Использование Общероссийского математического портала Math-Net.Ru подразумевает, что вы прочитали и согласны с пользовательским соглашением

http://www.mathnet.ru/rus/agreement

Параметры загрузки:

IP : 54.147 .182 .235

26 апреля 2023 г., 14:52:05 
ТЕОРЕТИЧЕСКАЯ

И МАТЕМАТИЧЕСКАЯ

ФИЗИКА

Том 119, № 3

июнь, 1999

(C) 1999 г.

В. Ф. Ковалев*

\section{РЕНОРМГРУППОВОЙ АНАЛИЗ \\ СИНГУЛЯРНОСТИ В ЗАДАЧЕ О САМОФОКУСИРОВКЕ ВОЛНОВОГО ПУЧКА}

Построением ренормгрупповых симметрий исследовано сингулярное решение краевой задачи для системы уравнений, описывающих самофокусировку волнового пучка. Получены новые аналитические зависимости, характеризующие пространственную эволюцию пучка с произвольным начальным профилем интенсивности в среде с кубичной нелинейностью. Детально проанализировано поведение гауссова пучка вплоть до момента возникновения особенности решения и предложена гипотеза, позволяюшая описать его структуру после появления сингулярности.

\section{1. ВВЕДЕНИЕ}

В оптике и физике плазмы хорошо известен эффект самофокусировки волнового пучка, когда его интенсивность лавинообразно нарастает в ограниченной области пространства (см., например, обзоры $[1,2])$. С математической точки зрения это явление соответствует возникновению (возможно, сглаженной) особенности в решении уравнений, используемых для описания пространственной эволюции пучка. Наиболее простой и универсальной математической моделью для анализа характерных особенностей эффекта самофокусировки является нелинейное уравнение Шредингера, для исследования сингулярных решений которого используются различные аналитические и численные подходы (см., например, книгу [3] и обзоры $[1,2,4,5]$. Каждому из этих подходов присуши свои ограничения, не позволяюшие провести анализ нелинейного уравнения Шредингера при произвольных краевых данных, поэтому разработка любого аналитического метода исследования его решений, в том числе и сингулярных, является по-прежнему актуальной.

В предлагаемой работе для построения и исследования сингулярных решений нелинейного уравнения Шредингера применяется метод, основанный на использовании особого класса симметрий, так называемых симметрий ренормгруппового типа. Для математических моделей, базирующихся на дифференциальных уравнениях, описание регулярных алгоритмов построения ренормгрупповых симметрий (РГС) можно найти в [6].

\footnotetext{
* Институт математического моделирования РАН, Москва, Россия. E-mail: kovalev@imamod.ru
} 
В недавних работах с помощью ренормгруппового метода изучались точные и приближенные аналитические решения более простых (по сравнению с нелинейным уравнением Шредингера) уравнений нелинейной геометрической оптики, не учитьвающих дифракционных эффектов [7-10]. Переход от уравнений нелинейной геометрической оптики к нелинейному уравнению Шредингера, которое записано во втором разделе в виде эквивалентной системы уравнений для интенсивности и производной эйконала пучка (уравнений нелинейной оптики волнового пучка в квазиоптическом приближении), не просто повышает порядок исходных уравнений, но наряду с параметром нелинейности вводит в рассмотрение новый параметр, обусловленный дифракцией волнового пучка. При малых значениях параметров, характеризуюших нелинейные и дифракционные эффекты, РГС для исходных уравнений построены в третьем разделе в виде бесконечных рядов по степеням этих параметров. Для среды с кубичной нелинейностью получены явные выражения для линейных по параметрам нелинейности и дифракции координат оператора РГС. Показано, что построенная РГС является симметрией Ли-Беклунда третьего порядка и в зависимости от вида распределения интенсивности пучка на границе среды оказывается либо точной, либо приближенной симметрией. Для цилиндрического волнового пучка оператор РГС Ли-Беклунда эквивалентен оператору точечной РГС, и для него конечные преобразования группы, дающие искомое решение краевой задачи, записываются в явном виде. Четвертый раздел посвящен анализу полученных решений краевой задачи для различных распределений интенсивности волнового пучка на границе среды. Детально исследована пространственная эволюция гауссова пучка, сопровождающаяся образованием особенности на оси. Показано, что за точкой особенности найденное решение является неоднозначным, и обсуждается возможный способ устранения этой неоднозначности, приводящий к существованию пространственной структуры пучка с осевой сингулярностью. В пятом разделе кратко сформулированы основные отличия ренормгруппового метода от других известных в настоящее время аналитических подходов к исследованию пространственной эволюции волнового пучка. В заключении рассмотрена возможность дальнейшего приложения как уже найденных, так и новых РГС, которые могут быть получены модификацией используемой математической модели.

\section{2. ИСХОДНЫЕ УРАВНЕНИЯ}

Для описания стационарной самофокусировки волнового пучка, распространяющегося вдоль оси $z$, в квазиоптическом приближении используется нелинейное уравнение Шредингера для комплексной амплитуды $E=A \exp (i k s)$ электрического поля (см., например, $[1])$, которое после введения интенсивности $I$ и производной эйконала $v$, связанных с амплитудой $A$ и эйконалом $s$ формулами

$$
I \equiv A^{2}=|E|^{2}, \quad \mathbf{v}=\nabla_{\perp} s
$$

сводится к следующим двум уравнениям, рассматриваемым ниже в качестве исходных:

$$
\begin{gathered}
v_{t}+v v_{x}-\alpha \varphi(n) n_{x}-\beta \partial_{x}\left(\frac{x^{1-\nu}}{\sqrt{n}} \partial_{x}\left(x^{\nu-1} \partial_{x}(\sqrt{n})\right)\right)=0 \\
n_{t}+n v_{x}+v n_{x}+(\nu-1) \frac{n v}{x}=0
\end{gathered}
$$


Здесь оператор $\nabla_{\perp}$ действует в плоскости, ортогональной к оси $z$, вектор $\mathbf{v}$ имеет единственную отличную от нуля компоненту $v$ в этой плоскости, $k=(\omega / c) \sqrt{\varepsilon_{0}}, \varepsilon_{0}-$ действительная часть линейной диэлектрической пронишаемости среды. В уравнениях $(2.2)$ использованы безразмерные переменные

$$
t=\frac{z}{d}, \quad x=\frac{r}{d}, \quad n=\frac{I}{I_{0}},
$$

где $d$ - характерный поперечный размер пучка, $I_{0}$ - максимальное значение интенсивности пучка на границе среды, параметры $\alpha=\gamma I_{0} / 2 \varepsilon_{0}$ и $\beta=1 / 2 k^{2} d^{2}$ определяют вклад процессов нелинейной рефракции и дифракции, $\nu=1$ для плоского (двумерного) волнового пучка и $\nu=2$ для трехмерного (осесимметричного) волнового пучка. Постоянная $\gamma$ возникает в качестве коэффициента при линейном вкладе в разложении нелинейной добавки $\varepsilon_{2}$ диэлектрической проницаемости среды в ряд по степеням интенсивности пучка, $\varepsilon_{2}=\gamma I+O\left(I^{2}\right)$. Для среды с кубичной нелинейностью этот вклад является единственным, а для другого типа нелинейности влияние среды учитывается посредством функции $\varphi(n)$, которая при этом отличается от единицы.

Уравнения (2.2) следует дополнить краевыми условиями, определяюшими структуру пучка на границе нелинейной среды (при $t=0$ ). Выбор этих условий в виде линейно изменяюшейся функции $v(0, x)$ и произвольной гладкой функции $n(0, x)$ :

$$
v(0, x)=V(x)=-\frac{x}{T}, \quad n(0, x)=N(x),
$$

задает на границе среды сфокусированный волновой пучок с произвольным гладким распределением интенсивности.

\section{3. РЕНОРМГРУППОВЫЕ СИММЕТРИИ И РЕШЕНИЕ КРАЕВОЙ ЗАДАЧИ}

При отыскании решения краевой задачи в данном исследовании ключевым моментом является построение РГС. При найденном инфинитезимальном операторе РГС искомое решение краевой задачи $(2.2),(2.4)$ получается из конечных преобразований группы, связывающих значение интенсивности и производной эйконала пучка в нелинейной среде с граничными значениями (2.4). Разнообразные способы построения РГС уже обсуждались в предшествуюших работах (см., например, [6]), в том числе и в применении к задачам нелинейной оптики [7-9]. Ниже мы будем следовать способу, близкому к изложенному в [9]. Опуская подробные выкладки и отсылая за необходимыми для понимания промежуточными результатами к приложению, изложим здесь только основную схему построения РГС и приведем окончательный результат.

Для построения РГС используются допускаемые исходными дифференциальными уравнениями (2.2) симметрии Ли-Беклунда, задаваемые каноническим оператором группы,

$$
X=f \partial_{v}+g \partial_{n} .
$$

Координаты $f$ и $g$ этого оператора находятся решением соответствуюших определяющих уравнений, выражаюших условия инвариантности системы $(2.2)$ относительно 
группы с оператором (3.1). Представим $f$ и $g$ в виде рядов по степеням параметров нелинейности $\alpha$ и дифракции $\beta$ :

$$
f=\sum_{i, j=0}^{\infty} \alpha^{i} \beta^{j} f^{(i, j)}, \quad g=\sum_{i, j=0}^{\infty} \alpha^{i} \beta^{j} g^{(i, j)}
$$

где коэффишиенты разложений $f^{(i, j)}, g^{(i, j)}$ являются функциями $t, x, v, n$ и производных от $v$ и $n$ по $x$ произвольного порядка. Подстановка в определяющие уравнения выражений (3.2) и приравнивание вкладов при одинаковых степенях $\alpha$ и $\beta$ порождают систему уравнений для коэффициентов $f^{(i, j)}$ и $g^{(i, j)}$. В приложении приведена система уравнений для нахождения вкладов $f^{0} \equiv f^{(0,0)}, g^{0} \equiv g^{(0,0)}$, не зависящих от $\alpha$ и $\beta$, и вкладов, линейных по параметрам нелинейности и дифракции $f^{1} \equiv \alpha f^{(1,0)}+\beta f^{(0,1)}$, $g^{1} \equiv \alpha g^{(1,0)}+\beta g^{(0,1)}$. Именно такими вкладами в бесконечные ряды (3.2) мы и ограничимся в дальнейшем, пренебрегая билинейными вкладами и вкладами второго и более высоких порядков:

$$
f=f^{0}+f^{1}+O\left(\alpha^{2}, \beta^{2}, \alpha \beta\right), \quad g=g^{0}+g^{1}+O\left(\alpha^{2}, \beta^{2}, \alpha \beta\right) .
$$

Это предположение оправдано в том случае, если либо координаты $f, g$ содержат только линейные по параметрам $\alpha$ и $\beta$ вклады, либо величины этих параметров малы. В последнем случае отбрасывание пропорциональных $O\left(\alpha^{2}, \beta^{2}, \alpha \beta\right)$ слагаемых означает, что получаемая симметрия является приближенной по указанным параметрам.

Найденные при решении определяющих уравнений координаты $f$ и $g$ зависят от набора произвольных функций (см. приложение, замечание после формулы (П.2)). Этот произвол устраняется с помошью сужения группы, при котором проверяются выполнение условия инвариантности

$$
f=0, \quad g=0
$$

на решении краевой задачи и соответствие этого условия заданным краевым данным (2.4). В результате приходим к искомому оператору РГС, координаты которого $f$ и $g$ для цилиндрического пучка $(\nu=2)$ в среде с кубичной нелинейностью $(\varphi=1)$ имеют Вид

$$
\begin{aligned}
f= & D_{x}\left\{\frac{1}{2 T^{2}}\left(x+v T\left(1-\frac{t}{T}\right)\right)^{2}-\right. \\
& \left.-\left(1-\frac{t}{T}\right)^{2}\left(\alpha n+\frac{\beta}{x \sqrt{n}} D_{x}\left(x D_{x} \sqrt{n}\right)\right)+S\right\}, \\
g= & \frac{1}{x} D_{x}\left\{(x n)\left[\frac{1}{T}\left(1-\frac{t}{T}\right)(x+v T-v t)-t S_{\chi}\right]\right\} .
\end{aligned}
$$

Здесь $D_{x}$ задает оператор полного дифференцирования по переменной $x$

$$
D_{x} \equiv \partial_{x}+\sum_{s=0}^{\infty}\left(v_{s+1} \partial_{v_{s}}+n_{s+1} \partial_{n_{s}}\right), \quad v_{s}=\frac{\partial^{s} v}{\partial x^{s}}, \quad n_{s}=\frac{\partial^{s} n}{\partial x^{s}}
$$


а функция $S$ зависит от переменной $\chi=x-v t$ :

$$
S(\chi)=\alpha N(\chi)+\frac{\beta}{\chi \sqrt{N(\chi)}} \partial_{\chi}\left(\chi \partial_{\chi}(\sqrt{N(\chi)})\right) .
$$

Аналогичные (3.5) формулы для координат $f, g$ оператора РГС для плоского пучка $(\nu=1)$ в среде с кубичной нелинейностью приведены в приложении.

Каноническому оператору ренормгруппы с координатами (3.5) эквивалентен следуюший оператор точечной ренормгруппы:

$$
\begin{aligned}
R= & {\left[\left(1-\frac{t}{T}\right)^{2}+t^{2} S_{\chi \chi}\right] \partial_{t}+\left[\frac{x}{T^{2}}+\frac{v}{T}\left(1-\frac{t}{T}\right)+S_{\chi}\right] \partial_{v}+} \\
& +\left[-\frac{x}{T}\left(1-\frac{t}{T}\right)+t S_{\chi}+v t^{2} S_{\chi \chi}\right] \partial_{x}+ \\
& +\left[\frac{2 n}{T}\left(1-\frac{t}{T}\right)-n t\left(1+\frac{v t}{x}\right) S_{\chi \chi}-\frac{n t}{x} S_{\chi}\right] \partial_{n}
\end{aligned}
$$

который служит инструментом для получения решений искомой краевой задачи. Последние возникают из конечных преобразований группы, связывающих значения интенсивности пучка $n$ и производной эйконала $v$ при $t>0$ с аналогичными величинами на границе $t=0$ нелинейной среды

$$
v(t, x)=\frac{x-\chi}{t}, \quad n(t, x)=N(\mu)\left(1-\frac{t}{T}\right)^{-1} \frac{\chi}{x} \frac{S_{\chi^{2}}}{S_{\mu^{2}}} .
$$

Здесь функции $\chi$ и $\mu$ определены через $t$ и $x$ с помошью соотношений

$$
x=\chi\left(1-\frac{t}{T}\right)\left(1+\frac{2 t^{2} S_{\chi^{2}}}{\left(1-\frac{t}{T}\right)^{2}}\right), \quad S(\mu)=S(\chi)+\frac{t^{2} S_{\chi}^{2}}{2\left(1-\frac{t}{T}\right)^{2}} .
$$

Подчеркнем, что при получении формул (3.9) не делалось никаких априорных предположений о пространственной структуре пучка в среде, и, следовательно, эти соотношения пригодны для пучков с произвольными гладкими краевыми данными (2.4). Единственное ограничение, делаюшее соотношения (3.9) не точными, а приближенными (за исключением нескольких избранных типов зависимостей $S(x))$, связано с малостью коэффициентов $\alpha$ и $\beta$. Эти условия используются при построении РГС в виде отрезка рядов (3.3), которая в этом смысле является приближенной.

\section{4. ПРОСТРАНСТВЕННАЯ СТРУКТУРА ВОЛНОВОГО ПУЧКА И ВОЗНИКНОВЕНИЕ ОСОБЕННОСТИ}

Рассмотрим с помошью формул (3.9) пространственную эволюцию цилиндрического пучка при нескольких различных вариантах распределения его интенсивности на гранище нелинейной среды. При этом особое внимание обратим на вопрос сушествования особенности решения (3.9). 
4.1. Пусть $S(x)$ является биномом

$$
S(x)=s_{0}+\frac{s_{2} x^{2}}{2}
$$

В этом случае соотношения (3.9) являются точным решением исходных уравнений (2.2) и справедливы при произвольных значениях параметров $\alpha$ и $\beta$. Такой вид $S(x)$ отвечает вполне определенной зависимости от координаты $x$ интенсивности пучка $N$ на границе среды, которая дается решением дифференциального уравнения второго порядка для функции $Q(x)=\sqrt{N(x)}$

$$
\beta\left(Q_{x x}+\frac{1}{x} Q_{x}\right)+\alpha Q^{3}-\left(s_{0}+\frac{s_{2} x^{2}}{2}\right) Q=0 .
$$

В приближении нелинейной геометрической оптики, т.е. при $\beta=0$, это уравнение описывает пучок с параболическим профилем. При $s_{2}=0$ и $s_{0}>0$ результаты численного решения уравнения (4.2) приводились в различных работах (см., например, [11-13]), посвященных исследованию поперечного распределения амплитуды самоканалированного пучка. Было показано сушествование гладких в нуле и спадающих на бесконечности локализованных решений. При $s_{2} \neq 0$ уравнение (4.2) также допускает существование локализованных, убывающих на бесконечности решений либо с монотонной (при $s_{2}>0$ ), либо с осциллируюшей, но спадаюшей степенным образом (при $s_{2}<0$ ), асимптотикой $[4,14]$.

Подстановка выражения (4.1) в (3.9) приводит к формулам, которые сохраняют вид зависимости $v$ и $n$ от поперечной координаты и по внешнему виду аналогичны соотношениям, возникаюшим в линейной оптике при учете дифракционных эффектов,

$$
\begin{aligned}
& v(t, x)=-\frac{x}{T} \frac{1-\frac{t}{T}-s_{2} t T}{\left(1-\frac{t}{T}\right)^{2}+s_{2} t^{2}} \\
& n(t, x)=N\left(\frac{x}{\sqrt{\left(1-\frac{t}{T}\right)^{2}+s_{2} t^{2}}}\right) \frac{1}{\left(1-\frac{t}{T}\right)^{2}+s_{2} t^{2}} .
\end{aligned}
$$

Непосредственно из вида (4.3) следует, что возникновение особенности на оси пучка возможно только для $s_{2} \leqslant 0$. При этом фокусировка пучка в точку при $t=T$ для $s_{2}=0$, т.е. для “таунсова" пучка $[11,13]$, имеет “чисто геометрический” характер, обусловленный кривизной фазового фронта пучка на границе среды. При $T \rightarrow \infty$ происходит самоканализация пучка без образования особенности. Для отрицательных $s_{2}$ интенсивность на оси пучка обрашается в бесконечность за конечное время $t_{f 1}=T /\left(1+\sqrt{-s_{2} T^{2}}\right)$ с характерным "взрывным" нарастанием интенсивности на оси пучка при подходе к сингулярности $n_{\text {axes }}=1 /\left(1-t / t_{f 1}\right)\left(1-t / t_{f 2}\right)$, где $t_{f 2}=T /\left(1-\sqrt{-s_{2} T^{2}}\right) ;$ при этом в точке особенности пересекаются все лучи пучка ${ }^{1)}$. В целом ситуация подобна той, которая наблюдается для параболического пучка в нелинейной геометрической оптике (см., например, [1, с. $37 ; 15$, с. 175]).

\footnotetext{
${ }^{1)}$ Направление луча задается нормалью к поверхности фазового фронта пучка в данной точке.
} 
4.2. Рассмотрим теперь эволюцию пучка с гауссовым начальным профилем интенсивности $N(x)=\exp \left(-x^{2}\right)$, для которого $S(x)$ принимает вид

$$
S(x)=\alpha \exp \left(-x^{2}\right)+\beta\left(x^{2}-2\right) .
$$

Поскольку при $\alpha=0$ эта функция имеет квадратичную зависимость от $x$ вида (4.1), то величина $\beta$ в формуле (4.4) в пределе $\alpha \rightarrow 0$ может быть произвольной, но не обязательно малой. Подстановка (4.4) в (3.9) приводит к формулам, определяющим пространственную структуру гауссова пучка в нелинейной среде,

$$
v(t, x)=\frac{x-\chi}{t}, \quad n(t, x)=e^{-\mu^{2}}\left(1-\frac{t}{T}\right)^{-1} \frac{\chi}{x} \frac{\left(\beta-\alpha e^{-\chi^{2}}\right)}{\left(\beta-\alpha e^{-\mu^{2}}\right)} .
$$

Здесь функции $\chi$ и $\mu$ определены через $t$ и $x$ с помошью соотношений

$$
\begin{aligned}
& \beta \mu^{2}+\alpha e^{-\mu^{2}}=\beta \chi^{2}+\alpha e^{-\chi^{2}}+\frac{2 t^{2} \chi^{2}}{\left(1-\frac{t}{T}\right)^{2}}\left(\beta-\alpha e^{-\chi^{2}}\right)^{2} \\
& x=Y(t, \chi) \equiv\left(1-\frac{t}{T}\right) \chi\left[1+\frac{2 t^{2}}{\left(1-\frac{t}{T}\right)^{2}}\left(\beta-\alpha e^{-\chi^{2}}\right)\right] .
\end{aligned}
$$

Из этих формул следует, что, в отличие от предыдушего примера, для гауссова пучка при $\alpha \neq 0$ вид зависимости $v$ и $n$ от поперечной координаты $x$ не только не сохраняется при изменении координаты $t$, но и становится качественно иной. В частности, равенство $v=0$ имеет место не только на оси пучка, но и в точке с координатой $x_{0}$,

$$
x_{0}^{2}=\ln \frac{\alpha t}{\beta t-\frac{1}{2 T}\left(1-\frac{t}{T}\right)} .
$$

Формула (4.7) задает кривую, на которой происходит поворот лучей пучка. Условие положительности $x_{0}>0$ ограничивает интервал по координате $t$, на котором определена кривая (4.7),

$$
\frac{T}{1+2 \beta T^{2}}<t<\frac{T}{1+2(\beta-\alpha) T^{2}}
$$

для $\alpha>\beta$ ограничений на величину $t$ сверху не существует.

Определяемая формулами (4.5), (4.6) зависимость $x$ от $\chi$, а следовательно, и зависимость $v$ от $x$, не является однозначной при всех значениях параметров $\alpha, \beta$ и координаты $t$. Неоднозначность появляется при выполнении двух условий на функцию $Y(\chi)$ :

$$
Y_{\chi}=0, \quad Y_{\chi \chi}=0 .
$$

Отсюда следует ограничение на параметры $\beta<\alpha$ и возникает квадратное уравнение для нахождения координаты точки на оси пучка, за которой проявляется неоднозначность. Наименьший положительный корень этого уравнения $t_{b 1}=T /(1+\sqrt{2(\alpha-\beta)} T)$ определяет ближайшую к гранище среды точку неоднозначности. Второй корень уравнения $t_{b 2}=T /(1-\sqrt{2(\alpha-\beta)} T)$ сушествует лишь при $T<T_{\min }=1 / \sqrt{2(\alpha-\beta)}$. 
Размер области неоднозначности вдоль оси пучка при $T<T_{\min }$ :

$$
\Delta t \equiv t_{b 2}-t_{b 1}=\frac{2 T^{2} \sqrt{2(\alpha-\beta)}}{1-2(\alpha-\beta) T^{2}}
$$

растет с уменьшением кривизны фронта пучка на входе в среду. При $T>T_{\min }$ области неоднозначности решения соответствует весь бесконечный интервал оси за точкой $t_{b 1}$.

Поперечный размер области неоднозначности $\Delta x$ при каждом фиксированном $t$ дается формулой

$$
\Delta x=-\frac{4 \alpha t^{2}}{1-\frac{t}{T}} \chi_{\max }^{3} e^{-\chi_{\max }^{2}},
$$

где величина $\chi_{\max }$ является точкой локального максимума, т.е. находится из первого условия (4.8), которое принимает вид

$$
\left(1-\frac{t}{T}\right)^{2}+2 t^{2}\left(\beta+\alpha\left(2 \chi_{\max }^{2}-1\right) e^{-\chi_{\max }^{2}}\right)=0 .
$$

Как следует из соотношений (4.10) и (4.11), величина $\Delta x$ при $t=t_{b 1}$ равна нулю и растет с ростом $t$ вплоть до $t=T$, а затем уменьшается до нуля при $t=t_{b 2}$ (если, конечно, $t_{b 2}>0$, т.е. $\left.T<T_{\min }\right)$. Наибольшего значения поперечный размер $\Delta x \rightarrow \infty$ достигает при $t \rightarrow T$, т.к. в этом пределе величина $\chi_{\max }$, как следует из соотношения (4.11), остается ограниченной, а $\Delta x$ растет как $1 /(1-t / T)$.

Из условий (4.8) следует, что в точке $t_{b 1}$ производная $(\partial v / \partial x)$ и интенсивность пучка $n$ на оси обрашаются в бесконечность (см. ниже формулы (4.13)), т.е. точка, за которой возникает неоднозначность решения, соответствует моменту появления сингулярности на оси пучка. Отметим, что координаты обеих сингулярных точек на оси пучка $t_{f 1}$ и $t_{b 1}$ могут быть получены из единой формулы

$$
t_{s}=\frac{T}{1+T \sqrt{-2 S_{\chi^{2}}(0)}} .
$$

Закон нарастания интенсивности на оси пучка при $t<t_{b 1}$, как и в предыдущем примере, при подходе к сингулярности носит “взрывной” характер $n_{\text {axes }}=1 /\left(\left(1-t / t_{b 1}\right) \times\right.$ $\left.\left(1-t / t_{b 2}\right)\right)$. В самой точке $t_{b 1}$ характер изменения производной эйконала $v$ и закон спадания интенсивности $n$ в малой окрестности оси определяются разложением функции $Y$ при $\chi \rightarrow 0:$

$$
\begin{aligned}
& v\left(t_{b 1}, x\right)=\frac{x}{t_{b 1}}\left(1-x^{-2 / 3}\left(\frac{1-\frac{t_{b 1}}{T}}{2 \alpha t_{b 1}^{2}}\right)^{1 / 3}\right), \\
& n\left(t_{b 1}, x\right)=\left(2 \alpha t_{b 1}^{2} x^{2}\left(1-\frac{t_{b 1}}{T}\right)^{2}\right)^{-1 / 3}, x \rightarrow 0 .
\end{aligned}
$$

В реальных физических условиях неограниченного роста интенсивности пучка не происходит, что указывает на некорректность использования математической модели на основе уравнения Шредингера с кубичной нелинейностью при подходе к точке особенности $t_{b 1}$ и за ней, т.е. в области неоднозначности. Для описания структуры пучка в этой области следует усложнить математическую модель (за счет учета эффектов 
насьшения нелинейности и нелинейного поглощения, отказа от параболического приближения и т.п.), что не является задачей данного исследования. Однако если принять модель среды с кубичной нелинейностью, то из решения (4.5), (4.6) можно получить физически разумный вариант ${ }^{2)}$ поведения пучка после момента возникновения особенности $t_{b 1}$. С этой целью ограничим значения параметра $\chi$ неравенством $\chi>\chi_{b}$, где величина $\chi_{b}$ определяется соотношением

$$
\begin{array}{ll}
\chi_{b}^{2}=\ln \frac{\alpha t^{2}}{\beta t^{2}+\frac{1}{2}\left(1-\frac{t}{T}\right)^{2}}, & t_{b 1}<t<T, \\
\chi_{b}=0, & 0 \leqslant t \leqslant t_{b 1} .
\end{array}
$$

Зависимость $x=Y(t, \chi)$ от $\chi$ при этом является однозначной во всей области изменения координаты $0<x<\infty$. Исключение из возможных значений параметра $\chi$ области $|\chi|<\chi_{b}$ с физической точки зрения эквивалентно предположению об отсутствии лучей, пересекающих ось пучка. В условиях существования особенности при $t_{b 1}<t<T$, когда интенсивность поля на оси пучка равна бесконечности, и, следовательно, бесконечным оказывается показатель преломления среды, это соответствует практически мгновенному повороту входяшего в ось луча (для которого $x=0, \chi=\chi_{b}, v=-\chi_{b} / t \neq 0$ ) или, другими словами, его трансформации в осевой луч (для которого $v=0, x=$ $\chi=0)$. При этом особенность на оси пучка, возникшая при $t=t_{b 1}$, сохраняется и поток энергии в нее продолжает нарастать вплоть до $t=T$, что позволяет говорить об образовании фокальной "нити” (ср. с [16], где для пространства большей размерности подобная картина пространственно-временных лучей получила название распределенного коллапса).

Для определения характера убывания интенсивности пучка при удалении от оси в области $t_{b 1}<t<T$ перейдем в формулах (4.5), (4.6) к пределу $\chi \rightarrow \chi_{b}$ и $x \rightarrow 0$ :

$$
\begin{gathered}
n(t, x)=\frac{e^{-\mu^{2}}}{\alpha e^{-\mu^{2}}-\beta} \frac{1-\frac{t}{T}}{2 t^{2} x}\left(\ln \left(\frac{\alpha}{\beta+\left(1-\frac{t}{T}\right)^{2} / 2 t^{2}}\right)\right)^{1 / 2}, \\
\beta \mu^{2}+\alpha e^{-\mu^{2}}=\left(\beta+\frac{\left(1-\frac{t}{T}\right)^{2}}{2 t^{2}}\right)\left(1+\chi_{b}^{2}\right), \quad x \rightarrow 0 .
\end{gathered}
$$

При $t \rightarrow T$ величина $\mu \rightarrow \chi_{m}=\sqrt{\ln (\alpha / \beta)}$ и вместо (4.15) возникает более простая формула

$$
n(T, x)=\frac{\sqrt{\beta}}{2 T \alpha x}, \quad x \rightarrow 0
$$

При удалении от оси пучка изменение интенсивности в области $t_{b 1}<t<T$ характеризуется не только медленной обратно пропорциональной зависимостью порядка $1 / x$, но и экспоненциальной зависимостью порядка $\exp \left(-\mu^{2}\right)$, где $\mu$ и $x$ связаны соотношениями (4.6). При $t \rightarrow T$ даже для малых удалений от оси, соответствующих $\chi>\chi_{b} \rightarrow \chi_{m}$,

\footnotetext{
2) Здесь указана одна из возможностей построения однозначного решения для волнового пучка за точкой особенности. Автор благодарен проф. В.П. Силину за обсуждение вопроса, связанного с анализом возможных вариантов построения однозначного решения в окрестности точки особенности.
} 
величина $\mu$ оказывается большой, что приводит к резкому убыванию интенсивности с ростом $x$, т.е. происходит концентрация пучка как целого. Для несфокусированного пучка с плоским фазовым фронтом $T \rightarrow \infty$ такая концентрация оказывается невозможной на конечных расстояниях $t<\infty$.

\section{5. ОБСУЖДЕНИЕ РЕНОРМГРУППОВОГО МЕТОДА}

Поскольку в настоящее время известны различные точные и приближенные аналитические подходы к решению задачи об эволюции волнового пучка, то представляется естественным сопоставить их с ренормгрупповым методом, указав только на основные характерные его отличия и не останавливаясь на детальном анализе конкретных частных решений. По сравнению с методом обратной задачи рассеяния, который использовался при построении одно- и $N$-солитонных решений ${ }^{3)}$ в задаче о стационарном распространении плоского светового пучка в среде с кубичной нелинейностью [3, 19], ренормгрупповой метод пригоден и для изучения эволюции цилиндрического светового пучка, а также при замене кубичной нелинейности среды нелинейностью произвольного вида.

Исследование нелинейного уравнения Шредингера методами стандартного группового анализа, которьй является составным элементом ренормгруппового метода, дает набор (пусть и достаточно большой) частных инвариантных решений [20-22], но не позволяет описать эволюцию пучка при произвольных краевых данных.

Возможность построения аналитического решения краевой задачи при отсутствии априорных предположений о структуре пучка (кроме предположения о малости коэффициентов нелинейности и дифракции) отличает ренормгрупповой метод от безаберрационного приближения $[1,12,15,23]$ и вариационного подхода $[14,24]$, а получаемые в ренормгрупповом методе формулы дают полное описание пространственной эволюции интенсивности и производной эйконала пучка в отличие от усредненного описания в методе моментов [25].

Найденная с использованием ренормгруппового метода картина пространственной эволюции пучка во всей области изменения переменных в предельных случаях согласуется с результатами, следующими из различных приближенных аналитических подходов. Детальному сравнению этих результатов будет посвящено отдельное исследование, здесь же отметим только, что указанное в данной работе условие появления сингулярности решения в виде равенства $\alpha=\beta$ дает величину критической мощности $P_{\text {кр }}=c \lambda^{2} \sqrt{\varepsilon_{0}} / 32 \pi^{2} \gamma$, совпадаюшую с той, которая получается из безаберрационного предела, хотя пространственная структура пучка оказывается иной. При этом часто используемая для характеристики пучка величина среднего радиуса $\left\langle x^{2}\right\rangle$, определенная как отношение второго момента функции $n$ к ее нулевому моменту при $\alpha=\beta$, не только не обрашается в нуль в точке особенности, как это следует из безаберрационного приближения, но даже возрастает при удалении от границы. Убывание $\left\langle x^{2}\right\rangle$ начинает наблюдаться, когда параметр $\alpha$ более чем вчетверо превосходит $\beta$, что соответствует

\footnotetext{
3) В случае произвольных краевых данных при отыскании решений, отличающихся от солитонных, использование метода обратной задачи рассеяния не позволяет точно описать их эволюцию и поэтому приходится прибегать к различным приближениям, базирующимся на теории возмущений $[4,17]$ или на асимптотических методах [18].
} 
критерию начала самофокусировки, следующему из метода моментов и вариационного подхода. Полученная в работах $[16,26-28]$ зависимость поля вблизи особенности $E \sim \sqrt{L(t) /\left(t_{s f}-t\right)}$ в целом также согласуется с найденным решением (4.5). Однако вместо медленно меняющейся функции $L(t)$, которая в $[16,26-28]$ имеет логарифмическую (либо двойную логарифмическую) зависимость, в формуле (4.5) присутствует медленная алгебраическая зависимость. Несовпадение может быть связано с более сложной формой фазового фронта вблизи оси пучка (см. формулу (4.13)), отличной от параболической формы в $[16,26,27]$.

\section{6. ЗАКЛЮЧЕНИЕ}

Подведем итог. В настоящей работе с помошью РГС построено приближенное (при малых значениях параметров, характеризующих нелинейные и дифракционные эффекты), а для краевых данных специального вида точное аналитическое решение краевой задачи об эволюции волнового пучка в нелинейной среде. Для различных вариантов краевых данных получены условия существования сингулярного решения, найдены координаты сингулярных точек и определен закон нарастания интенсивности пучка на оси при приближении к особенности. Показано, что для гауссова пучка за точкой сингулярности возникает область неоднозначности, и сформулирована гипотеза, позволяющая продолжить решение за точку особенности. Отмечено, что при этом решение сохраняет осевую особенность в распределении интенсивности по поперечной координате, и указан характер этой особенности.

Возможности ренормгруппового метода не исчерпываются перечисленными выше конкретными физическими результатами по самофокусировке цилиндрического пучка. Одним из вариантов дальнейшего использования уже найденных РГС является исследование пространственной эволюции пучков с более сложным начальным распределением интенсивности, приводящим, например, к образованию мелкомасштабных структур, характерной чертой которых является одновременное появление особенностей не только на оси пучка, но и в точках с $x \neq 0$. При этом может потребоваться уточнение найденных приближенных формул для РГС путем учета квадратичных, билинейных и вкладов более высокого порядка в разложении (3.2) по степеням параметров $\alpha$ и $\beta$.

Другая возможность использования ренормгруппового метода связана с изменением математической модели среды, базирующейся на нелинейном уравнении Шредингеpa. C точки зрения более простой модели нелинейной геометрической оптики [9] приведенные здесь результаты являются одним из примеров такого изменения, позволившего учесть дифракционные эффекты наряду с нелинейностью среды. В зависимости от конкретной постановки физической задачи другие модификации модели заключаются, например, в рассмотрении механизмов нелинейности среды, учитывающих эффекты насышения и релаксации, добавлении линейного и нелинейного поглошения, отказе от параболического приближения. Эти возможности ренормгруппового метода расширяют область применимости результатов, которые могут быть получены на его основе, в том числе для интерпретации сильнонелинейных физических явлений, наблюдаемых при распространении мощного лазерного излучения в оптике и плазменной физике (см., например, [5]). Универсальность метода позволяет надеяться, что реализованный в данной работе ренормгрупповой анализ сингулярности может быть также проведен для ряда других математических моделей, имеюших общефизическое значение. 
Благодарности. Автор выражает благодарность проф. Д.В. Ширкову за постоянную поддержку и плодотворные дискуссии и д.ф.м.н. Н. В. Змитренко за обсуждение полученных результатов. Работа выполнена при финансовой поддержке РФФИ (проект № 96-15-96030 и проект № 96-01-01297).

\section{ПРИЛОЖЕНИЕ}

Здесь приводится система линейных уравнений в частных производных первого порядка для вычисления координат $f^{0}, g^{0}$ и $f^{1}, g^{1}$ канонического оператора (3.1):

$$
\begin{aligned}
& L_{0} f^{0}=0, \quad L_{1} g^{0}+L_{2} f^{0}=0, \\
& L_{0} f^{1}+D_{t}^{1} f^{0}-\alpha\left(\varphi D_{x}+\varphi_{n} n_{1}\right) g^{0}-\beta\left\{B_{n} g^{0}+\right. \\
& \left.\quad+B_{n_{1}}\left(D_{x} g^{0}\right)+B_{n_{2}}\left(D_{x}^{2} g^{0}\right)+B_{n_{3}}\left(D_{x}^{3} g^{0}\right)\right\}=0, \\
& L_{1} g^{1}+D_{t}^{1} g^{0}+L_{2} f^{1}=0, \quad B=D_{x}\left(\frac{D_{x}\left(x D_{x}(\sqrt{n})\right)}{x \sqrt{n}}\right) .
\end{aligned}
$$

Входящие в эти уравнения дифференциальные операторы имеют вид

$$
\begin{aligned}
& D_{t}^{0}=\partial_{t}-\sum_{s=0}^{\infty}\left[D_{x}^{s}\left(v v_{1}\right) \partial_{v_{s}}+\left(D_{x}^{s+1}(n v)+D_{x}^{s}\left(\frac{n v}{x}\right)\right) \partial_{n_{s}}\right] \\
& D_{t}^{1}=\sum_{s=0}^{\infty}\left[D_{x}^{s}\left(\alpha \varphi n_{1}+\beta B\right)\right] \partial_{v_{s}}, \quad D_{x}=\partial_{x}+\sum_{s=0}^{\infty}\left[v_{s+1} \partial_{v_{s}}+n_{s+1} \partial_{n_{s}}\right], \\
& L_{0}=D_{t}^{0}+v D_{x}+v_{1}, \quad L_{1}=L_{0}+\frac{v}{x}, \quad L_{2}=n D_{x}+n_{1}+\frac{n}{x} .
\end{aligned}
$$

Решения линейных дифференциальных уравнений для функций $f^{i}$ и $g^{i}$ содержат произвольные функции $F^{i}$ и $G^{i}$, зависящие от бесконечного набора инвариантов оператора $v D_{x}+D_{t}^{0}$. Для функций $F^{0}$ и $G^{0}$ используется наиболее простое представление, которое позволяет записать следуюшие формулы для координат $f^{0}$ и $g^{0}$ :

$$
\begin{aligned}
f^{0}= & \frac{1}{T^{2}}\left(x+v T\left(1-\frac{t}{T}\right)\right)\left(1+v_{1} T\left(1-\frac{t}{T}\right)\right), \\
g^{0}= & \frac{n}{T}\left(1-\frac{t}{T}\right)\left(1+v_{1} T\left(1-\frac{t}{T}\right)\right)+ \\
& +\frac{1}{T}\left(n_{1}+\frac{n}{x}\right)\left(1-\frac{t}{T}\right)\left(x+v T\left(1-\frac{t}{T}\right)\right) .
\end{aligned}
$$

Непосредственной подстановкой (П.3) в (3.4) можно убедиться, что функции $f^{0}$ и $g^{0}$ удовлетворяют условиям инвариантности при произвольных краевых данных вида (2.4).

Используя формулы (П.3) в уравнениях для функций $f^{1}$ и $g^{1}$, запишем эти уравнения в более простом виде для среды с кубичной нелинейностью $(\varphi(n)=1)$ :

$$
\begin{gathered}
L_{0}\left(f^{1}+\left(1-\frac{t}{T}\right)^{2}\left(\alpha n_{1}+\beta B\right)\right)=0 \\
L_{1} g^{1}+\frac{1}{x} D_{x}\left[(x n) D_{x}\left(\alpha N(\chi)+\frac{\beta}{\chi \sqrt{N}}\left(\chi(\sqrt{N})_{\chi}\right)_{\chi}\right)\right]=0 .
\end{gathered}
$$


Решение уравнений (П.4) приводит после операции сужения группы к формулам (3.5).

Аналогичным образом находятся решения дифференщиальных уравнений для функций $f^{i}, g^{i}$ и в случае плоского волнового пучка. Опуская промежуточные формулы, приведем окончательный результат в виде линейных по параметрам $\alpha$ и $\beta$ координат оператора РГС $f, g$ для плоского пучка $(\nu=1)$ в среде с кубичной нелинейностью $(\varphi=1)$ :

$$
\begin{aligned}
f= & D_{x}\left\{\frac{1}{2 T^{2}}\left(x+v T\left(1-\frac{t}{T}\right)\right)^{2}-\alpha n\left(1-\frac{t}{T}\right)^{2}+\right. \\
& +\alpha \frac{n}{v_{1} T}\left(1-\frac{t}{T}+\frac{1}{v_{1} T}\right) \ln n-\alpha \frac{n}{v_{1} T}\left(1-\frac{t}{T}\right)- \\
& \left.-\beta\left(1-\frac{t}{T}\right)^{2} \frac{1}{\sqrt{n}} D_{x}\left(D_{x}(\sqrt{n})\right)+\beta \frac{1}{\sqrt{N}}(\sqrt{N})_{\chi \chi}\right\}, \\
g= & D_{x}\left\{\frac{n}{T}\left(1-\frac{t}{T}\right)(x+v T-v t)-\alpha T N N_{\chi} \ln N+\right. \\
& +\alpha \frac{1}{T}\left[\frac{n n_{1}}{v_{1}^{2}}\left[\frac{\ln n}{v_{1} T}+\left(1-\frac{t}{T}+\frac{1}{v_{1} T}\right)(\ln n-1)\right]-\right. \\
& \left.-\frac{n^{2} v_{2}}{v_{1}^{3}}\left[\frac{2 \ln n-1}{v_{1} T}+\left(1-\frac{t}{T}+\frac{1}{v_{1} T}\right)\left(\ln n-\frac{3}{2}\right)\right]\right]- \\
& \left.-\beta \operatorname{tn}\left(\frac{(\sqrt{N})_{\chi \chi}}{\sqrt{N}}\right)_{\chi}\right\} .
\end{aligned}
$$

\section{Список литературы}

[1] С. А. Ахманов, А. П. Сухоруков, Р. В. Хохлов. УФН. 1967. Т. 93. Вып. 1. С. 19.

[2] В. Н. Луговой, А. М. Прохоров. УФН. 1973. Т. 111. Вып. 2. С. 203.

[3] В.Е. Захаров, С.В. Манаков, С.П. Новиков, Л. П. Питаевский. Теория солитонов: Метод обратной задачи. М.: Наука, 1980.

[4] В. А. Андреев. Тр. ФИАН им. П. Н. Лебедева. 1991. Т. 211. С. 3.

[5] L. Berge. Phys. Rep. 1998. V. 303. № 5, 6. P. 260.

[6] V.F. Kovalev, V. V. Pustovalov, D. V. Shirkov. J. Math. Phys. 1998. V. 39. P. 1170.

[7] В. Ф. Ковалев, В. В. Пустовалов, С. И. Сенашов. Дифф. уравнения. 1993. Т. 29. С. 1751.

[8] V.F. Kovalev. J. Nonlinear Math. Phys. 1996. V. 3. P. 351.

[9] В. Ф. Ковалев. ТМФ. 1997. Т. 111. № 3. С. 369.

[10] V. F. Kovalev, D. V. Shirkov. J. Nonlinear Opt. Phys. and Materials. 1997. V. 6. № 4. P. 443.

[11] R. Chiao, E. Garmire, G. Townes. Phys. Rev. Lett. 1964. V. 13. P. 479; 1965. V. 14. P. 1056; 1966. V. 16. P. 347.

[12] В. И. Таланов. Письма в ЖЭТФ. 1965. Т. 2. № 5. С. 218.

[13] З. К. Янкаускас. Изв. вузов. Радиофизика. 1966. Т. 9. С. 412.

[14] D. Wood. Stud. Appl. Math. 1984. V. 71. № 2. P. 103.

[15] А.В. Гуревич, А. Б. Шварцбург. Нелинейная теория распространения радиоволн в ионосффере. М.: Наука, 1973.

[16] С. Н. Власов, В. И. Таланов. Распределенный волновой коллапс в модели нелинейного уравнения Шредингера. В сб.: Нелинейные волны: динамика и эволюция. Отв. ред. А.В. Гапонов-Грехов, М. И. Рабинович. М.: Наука, 1989. С. 218.

[17] R. H. Enns, S.S. Rangnekar. Can. J. Phys. 1985. V. 63. № 5. P. 632.

[18] В. Е. Захаров, С. В. Манаков. ЖЭТФ. 1976. Т. 71. № 1. С. 203.

3 Теоретическая и математическая физика, т. 119, № 3, 1999 г. 
[19] В. Е. Захаров, А. Б. Шабат. ЖЭТФ. 1971. Т. 61. № 1(7). С. 118.

[20] В.Б. Таранов. Группы преобразований и инвариантные решения нелинейного уравнения Шредингера. Препринт КИЯИ-80-15. Киев: КИЯИ, 1980.

[21] L. Gagnon, P. Winternitz. J. Phys. A. 1988. V. 21. P. 1493; 1989. V. 22. P. 469; 1992. V. 25. P. 4425; Phys. Rev. A. 1989. V. 39. № 1. P. 296.

[22] S. Kumei. J. Math. Phys. 1977. V. 18. P. 256.

[23] С. А. Ахманов, А. П. Сухоруков, Р. В. Хохлов. ЖЭТФ. 1966. Т. 50. № 6. С. 1537.

[24] D. Anderson, M. Bonnedal. Phys. Fluids. 1979. V. 22. № 1. P. 105.

[25] С.Н. Власов, В.А. Петрищев, В.И. Таланов. Изв. вузов. Радиофизика. 1971. Т. 14. № 9. C. 1353.

[26] С. Н. Власов, Л. В. Пискунова, В. И. Таланов. ЖЭТФ. 1978. Т. 75. № 5(11). С. 1602.

[27] B. J. LeMesurier, G. C. Papanicolaou, C. Sulem, P.L. Sulem. Physica D. 1988. V. 32. P. 210.

[28] N. E. Kosmatov, V.E. Zakharov, V. F. Shvets. Physica D. 1991. V. 52. P. 16.

Поступила в редакцию 13.X.1998 г. 